\title{
Design of Cloud Clothing System Based on Haar Algorithm
}

\author{
Jinhuan Wang*, Chun Jiao, Shuai Xv, Fuzhou Li \\ Intelligent Science and Information Engineering College \\ Xi'an Peihua University \\ Xi'an, China \\ *Email: 360635476@qq.com
}

\begin{abstract}
With the rapid development of network technology, People are frequently unsatisfied with the online shopping, especially for the online shopping of clothes. For solve the problem, a cloud dressing system based on Haar algorithm is raised. It matches the collected data with Haar algorithm, endows the rotation angle to the model of the clothes, overlays the collected image and the modified image and finally displays those images on the screen. Then, we can obtain the effect of putting on the clothes, bringing great convenience to people. The test has verified its excellent performance.
\end{abstract}

Keywords: Haar algorithm; Target classifier; Cloud server; $3 D$ model

\section{INTRODUCTION}

Nowadays, online shopping has become a common consumption means of people in daily life. However, while you find a cloth style you like during online shopping, you do not how it will look like when you put it on. In the past, only buy the cloth put it on, can you know whether it is beautiful and fit. The project well solves such confusion during online shopping and brings great

convenience to people. As far as sellers are concerned, the project reduces the return and turnover rate and the investment in customer service; As far as consumers are concerned, the project allows consumers to directly see the effect of cloth you will put on through the screen, avoiding the troubles of exchanging products and the extra postage, which saves both time and money.

\section{II.HAAR ALGORITHM}

The characteristic value of Haar algorithm [6] reflects the gray change of the image. The characteristic of Haar are divided into three categories, including edge characteristic, linear characteristic, central characteristic and diagonal characteristic. Characteristic template is obtained through the combinations of three characteristics. There are only black and white rectangles in the characteristics template. The characteristic value of the template is the value obtained by subtracting the sum of black rectangle pixels from the sum of white rectangle pixels. The examples are as follows:
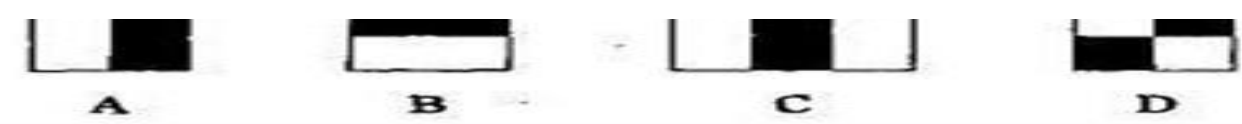

Figure 1 The image of the characteristic value of Haar algorithm

The calculation formula of the characteristic value of model A, B, D in the figure 2 is v=Sum white -Sum black. For ensuring the consistent number of pixels in two rectangle areas, the sum of black rectangle pixels in $\mathrm{C}$ model should be multiplied by 2 , so the formula should be $\mathrm{v}=$ Sum white $-2 *$ Sum black.

It can obtain many characteristics during the calculation by adjusting the size and position of the template. The values of those characteristics are called characteristic value. Because of the oversize of the quantity of characteristics, the integral graph in the figure 3 can be used in calculation.

The integral graph is a two-dimensional array with the same size of the image. In the array, the value of $(x, y)$ position is the sum of pixel value in the original image from $(0,0)$ to $(x, y)$. (a)

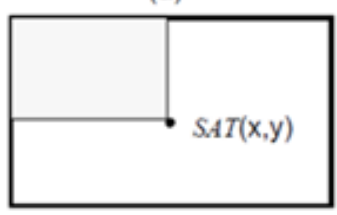

(b)

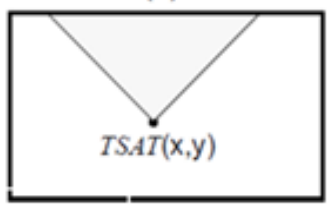

(c)

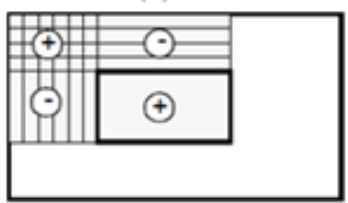

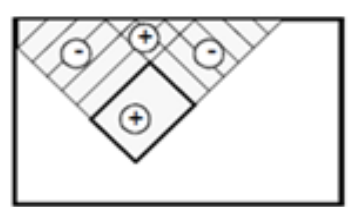

Figure 2. The integral graph

The calculation formulas of the haar characteristic shown in figure a and $\mathrm{c}$ are as follows:

$$
\operatorname{SAT}(x, y)=\sum_{x^{\prime} \leq x, y^{\prime} \leq y} I\left(x^{\prime}, y^{\prime}\right)
$$

$$
\operatorname{SAT}(x, y)=\operatorname{SAT}(x, y-1)+\operatorname{SAT}(x-1, y)+I(x, y)-\operatorname{SAT}(x-1, y-1)
$$




$$
\operatorname{RecSum}(r)=\operatorname{SAT}(x-1, y-1)+\operatorname{SAT}(x+w-1, y+h-1)-\operatorname{SAT}(x-1, y+h-1)-\operatorname{SAT}(x+w-1, y-
$$

SAT is the value of the integral graph. Rec Sum is the sum of the area at $(\mathrm{x}, \mathrm{y})$ with the length and width of $(\mathrm{w}, \mathrm{h})$.

The haar characteristic can be calculated after obtaining the Rec Sum.

$$
\begin{array}{r}
R S A T(x, y)=\operatorname{RSAT}(x-1, y-1)+\operatorname{RSAT}(x+1, y-1)+I(x, y)-R S A T(x, y-2)+I(x, y)+I(x, y-1) \\
\operatorname{RecSum}(r)=\operatorname{RSAT}(x-h+w, y+w+h-1)+\operatorname{RSAT}(x, y-1)-\operatorname{RSAT}(x-h, y+h-1)-R S A T(x+ \\
w, y+w-1)
\end{array}
$$

\section{A. Target Haar Classifier}

The objectives for training cascade classifier are to make the detection results more accurate, which involves another system of the Haar classifier, called detection system. It takes a big picture in reality as input, carries out the multiregional and multi-scale detection for the pictures, inputs the pictures and obtains a large number of sub window images which will be ceaselessly screened, abandoned, or allowed by each node of sieving cascade classifier. Its structure is shown in figure 3.

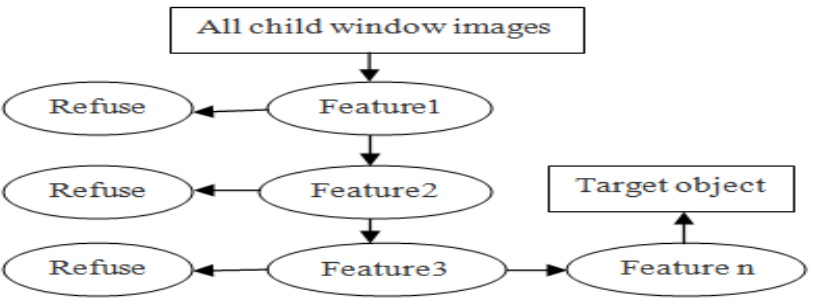

Figure 3. Target haar classifier

\section{B. 3D Model of the Cloud Dressing System}

3D model: The modification of 3D model is realized through the application of the Direct 3D ${ }^{[8]}$ interface. Descriptors a structure is used to tell Shaders where to find resources and how to explain resource data. It can create multiple descriptors for the same resource and send them to different stages with different means. Root Signatures has defined the data (resource) visited by Shader. Root Signatures is similar to a list of parameters of a function. Shader is the function and the type of data visited by Shader is the list of parameters.

\section{GENERAL DESIGN OF CLOUD DRESSING SYSTEM}

The system identifies the attempt of material object on the cloud platform with computer technology and certain algorithms. The attempt of most material objects purchased online can be verified through the technology. It changes the conventional habits of try-on and the experiential attempt of products purchased online and provides people with brand new ideas and means of shopping, bringing great convenience to people. It is an attempt of the popularization and application of intelligent recognition technology in daily life with profound significance and effect on the society and the daily life of people. Especially it will bring certain social and economic benefits to the social development dominated by the information. General structure of cloud dressing system is shown in figure 1 :

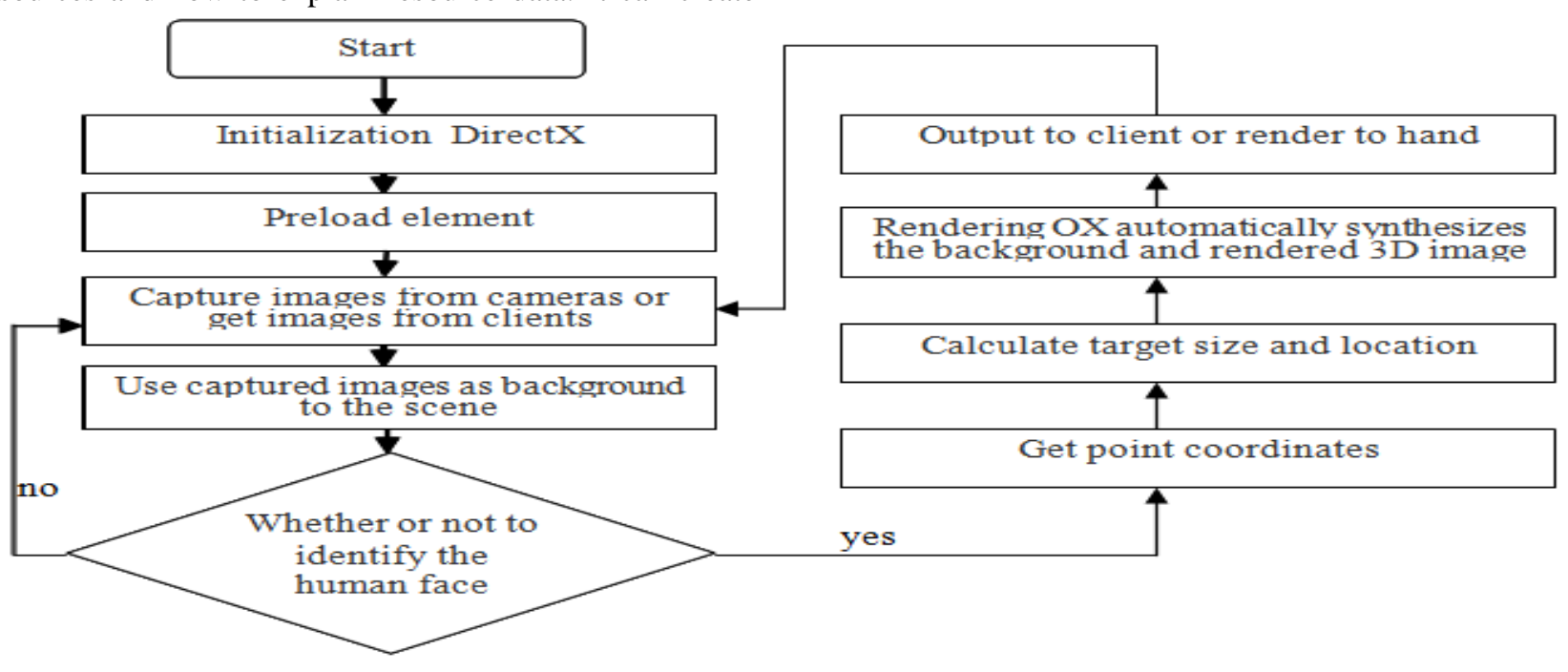

Figure 4. General structure of system

IV. SYSTEM TEST AND ANALYSIS

It gets the brightness of some points in the image through the grid average sampling, judges the light and shade of the object according to the brightness and determines the location of the light source based on the image background.

Cloud servers ${ }^{[9]}$ receive the data on clothes submitted 
by the seller (photo, size and others), construct the 3D model of clothes and output some parameters about clothing and human fitness for the downloading at the client end. The client needs to synthesize the animations of the clothes and modify the model and synthetics combining the parameters.

Specific Steps: (1) Capture images from the camera or client end;(2) Recognize the face and shoulder from the image; (3) Recognize specific objects, such as face, based on the machine learning [10] and Haar algorithm; Carry out the modification with DirectX. Carry out the modification of graphic elements by using the graphics card through the DirectX 12; (4) DirectX 12 modifies the OM within the line pipe and directly synthesize the image; Output the image or draw it on hand.
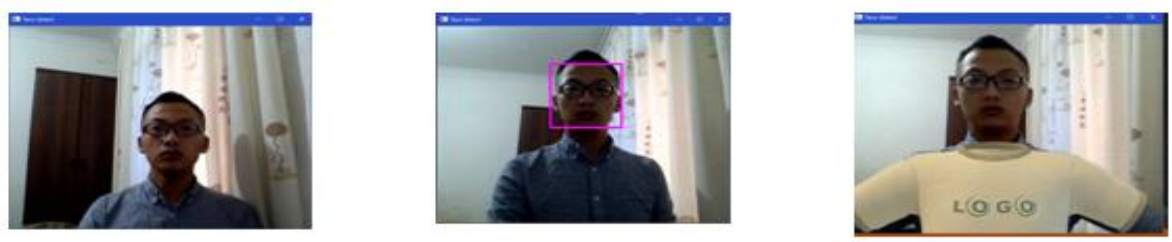

Figure 5. Image capture, Image recognition, Image synthesis

\section{V.CONCLUSIONS}

With the aid of computer software to establish the human body and the model of the human face using Haar algorithm to match, related data were collected through hardware launch laser measure the depth of field, combined with the light model source direction, object of light and shade and the matching results of human body model, comprehensive, determine the rotation Angle of the human body the rotation Angle and then will give clothing models, will obtain the figure of the rendering. The image is superimposed with the image collected, and the effect is obtained through the display screen.

\section{ACKNOWLEDGMENT}

This work is supported by 2018 Xi'an Peihua University Research Project" Design of English word memory Based on WeChat Small Program"(NO.PHKT18098) and 2017 Xi'an Peihua University Teaching Reform Research Project (No.PHZ1701), all support is gratefully acknowledged.

\section{REFERENCE}

[1] Ma Ying zhe Research on multi view face detection technology [J]. Liaoning Technical University. 2010.6.1-63.

[2] Zhang Jingjing Research on face detection and face feature positioning technology $[\mathrm{J}]$. GuangXi University for Nationalities. 2010.4.1.1-65.

[3] Duan Jin. Research on Some Problems in Automatic Face Recognition. [M].2004.10.1.1-133.

[4] Tomas Akenine-Moller/Eric Haines/Naty Hoffman, Real-Time Rendering third Edition[M], K Peters/CRC Press,2008.7

[5] Frank Luna,Introduction to 3D Game Programming with DirectX 12[M],Mercury Learning \& Information, 2015.12

[6] www.cnblogs.com/ello/archive/20, Brief analysis of face detection--Haar classifier,2012.4.28.

[7] Wang Qqingweim, Ying Zily. A face detection algorithm based on Haar-Like $\mathrm{T}$ characteristic $[\mathrm{J}]$. Fuzzy recognition and artificial intelligence.2015.1,28 (1),35-41.

[8] Jason Zink/Matt Pettineo/Jack Hoxley, Practial Rendering \& Computation with Direct 3D 11[M],A K Peters/CRC Press, 2011.7

[9] www.cloud.tencent.com/document/product/560/10419, Application Scenario - Cloud Server,2016.11

[10] Christopher Bishop, Pattern Recognition and Machine Learning[M], Springer, 2007.10 